\title{
Structure and Virulence of Pseudomonas Aeruginosa Bacteriophages
}

O.Sokolova $^{*}$, M.Filchikov $^{* *}$, E.Pechnikova ${ }^{* * *}$, K.Miroshnikov $^{* *}$

*Moscow State University, Dept Biology, Moscow, 119991

**Institute of Bioorganic Chemistry RAS, Moscow, 119997

***Institute of Crystallography RAS, Moscow, 119333

We used the single-particle cryo-electron microscopy and three-dimensional (3D) structural approach to solve the 3D structures of several bacteriophages that infects Pseudomonas aeruginosa. These gram-negative bacteria are frequently pathogenic in humans, and can cause severe diseases such as nephropathy, fibrosis and inflammation. Therefore, they have high potential for phage therapy $[1,2]$.

We calculated the 3D reconstructions of the capsids of the following bacteriophages: SN, KMV, $\mathrm{YuA}$ at the resolution $35 \AA$ and better (table 1). The capsids of all phages except $\mathrm{YuA}$ represent the regular icosahedrons with a triangulation $\mathrm{T}=9(\mathrm{SN})$ and $\mathrm{T}=7(\mathrm{KMV})$. YuA bacteriophage represents the elongated icosahedron with the C5 type of symmetry.

All phages possess the unique surface architecture of their capsids with prominent décor elements (Fig. 1, A-C). Using the polyclonal antibodies directed against structural components: pg22 of the phage SN, pg47 of the phage KMV and pg63 of the phage YuA we were able to demonstrate the surface distribution of these structural proteins (Fig.2, A-C).

To reveal the structural principles of phage virulence, we used the electron tomography as a powerful tool to study the interactions of phages with the wild type and mutant $P$. aeruginosa. The tomographic low-resolution reconstructions explained the specificity while binding of phages to bacteria cell surface for initiation of infection.

\section{References}

[1] Shaburova et al., Genetika 44, (2008) 713-716

[2] Krylov et al., Can. J. Microbiol. 30 (1984) 758-762

[3] Authors thank Prof. Nikolay Kiselev for helpful discussions and Dr. Alexander Vasiliev for help with usage of FEI Titan G2 electron microscope. 
TABLE 1. Parameters for the 3D reconstruction.

\begin{tabular}{lllllll}
\hline Phage & Systematics & $\begin{array}{l}\text { No of } \\
\text { images }\end{array}$ & Resolution & No of particles & $\begin{array}{l}\text { Type of } \\
\text { simmetry }\end{array}$ & $\begin{array}{l}\text { Resolution of } \\
\text { reconstruction }\end{array}$ \\
\hline SN & Myoviridae & 56 & $4.5 \AA$ & 645 & ICOS, C5 & $25 \AA$ \\
\hline KMV & Podoviridae & 126 & $3.5 \AA$ & 9320 & C5 & $35 \AA$ \\
\hline YuA & Siphoviridae & 54 & $3.5 \AA$ & 3142 & C5 & $35 \AA$ \\
\hline
\end{tabular}
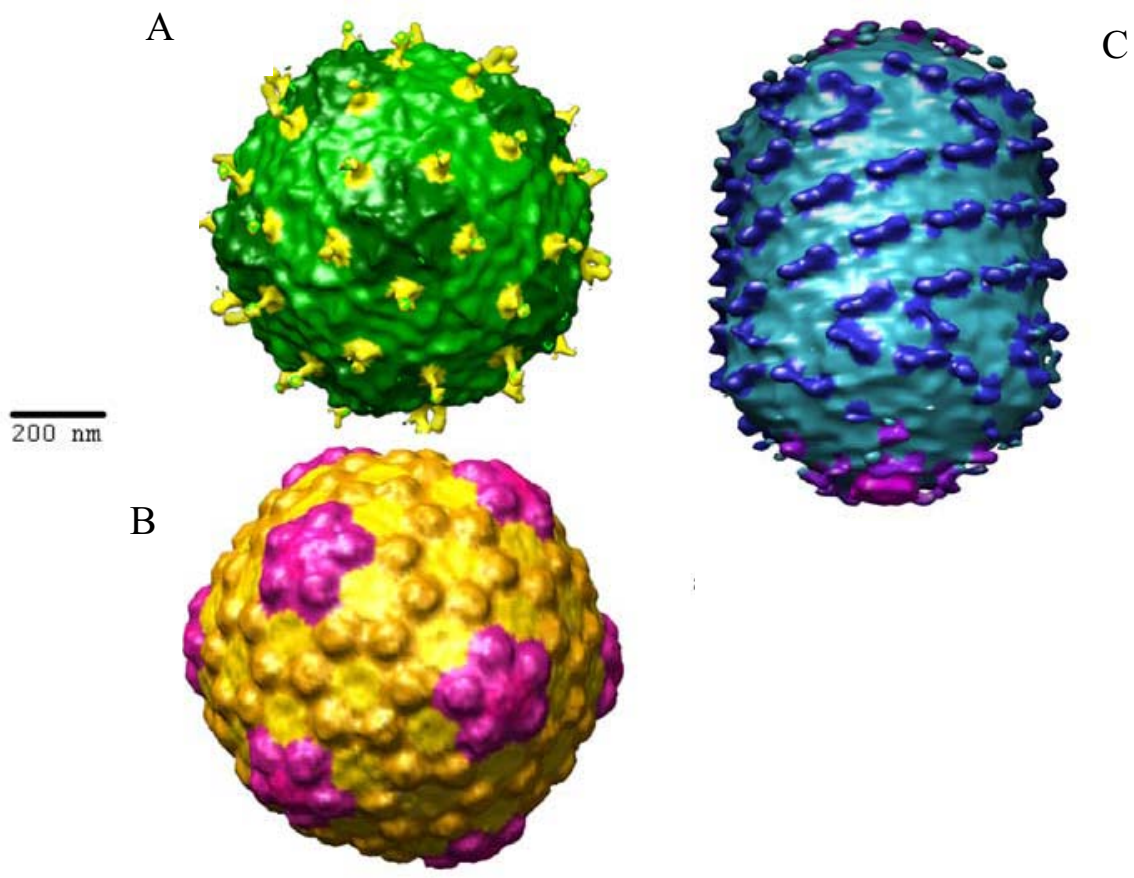

FIG. 1. 3D reconstructions of capsids of phages: (A) KMV; (B) SN; (C) YuA.

A

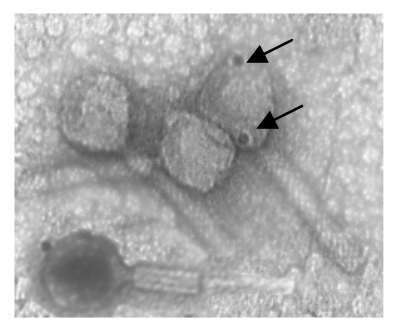

B

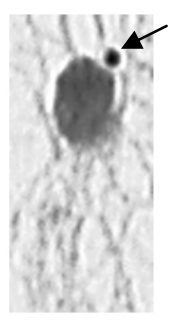

$\mathrm{C}$

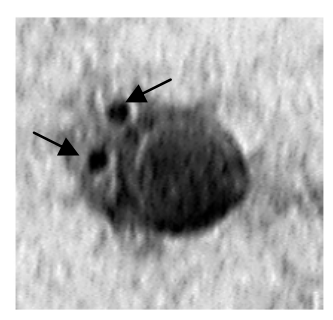

FIG. 2. Distribution of gold labeled antibodies (arrows) against (A) pg22; (B) pg63; (C) pg 57 